\title{
Aesthetic Treatments in Cancer Patients
}

\author{
Ilaria Proietti ${ }^{1}$ \\ Nevena Skroza (D) \\ Alessandra Mambrin' \\ Federica Marraffa ${ }^{2}$ \\ Ersilia Tolino' \\ Nicoletta Bernardini $(\mathbb{D})^{\prime}$ \\ Anna Marchesiello' \\ Giovanni Rossi' \\ Salvatore Volpe' \\ Concetta Potenza' \\ 'Dermatology Unit "Daniele Innocenzi”, \\ Department of Medical-Surgical Sciences \\ and Biotechnologies, Sapienza University \\ of Rome, Fiorini Hospital, Polo Pontino, \\ Terracina, 04019, Italy; ${ }^{2}$ Department of \\ Clinical Internal, Anaesthesiologic and \\ Cardiovascular Sciences, Sapienza \\ University of Rome, Rome, Italy
}

Correspondence: Ilaria Proietti Dermatology Unit "Daniele Innocenzi", Department of Medical-Surgical Sciences and Biotechnologies, Sapienza University of Rome, Fiorini Hospital, Polo Pontino, Terracina, 04019, Italy

Email ilaria.proietti@uniromal.it

\begin{abstract}
Cancer patients are experiencing an increase in overall survival as a consequence of earlier diagnosis and newer effective anticancer therapies. However, cancer survivors often face long-term consequences from their original cancer diagnosis and long-term sequelae of anticancer treatment. Maintaining patients' quality of life is of paramount importance and this can be accomplished by a multidisciplinary treatment approach, including aesthetic treatments to improve patients' body image and positively impact their quality of life. In this perspective, we will discuss the importance of aesthetic treatments in cancer patients. In addition, we will summarise the data available regarding the use of several aesthetic treatments such as fillers, botulinum toxin and laser use in cancer patients, their safety, their efficacy, and the specific precautions that need to be implemented in this particular subset of cancer patients.
\end{abstract}

Keywords: aesthetic treatment, cancer patient, fillers, botulinum toxin, laser therapy

\section{Introduction}

The survival rate of cancer patients has significantly increased due to early diagnosis and the efficacy of available treatments. However, there is a new concept emerging which explores what being a cancer patient actually entails, where cancer is no longer solely considered an acute disease, but an increasingly chronic illness that needs to be cured.

A noticeable consequence of the higher survival rate mentioned above, is an increase in the life expectancy of cancer patients, which in turn leads to a prolongation of the patients' coexistence with the collateral effects or problems related to the disease or its treatment. In other words, extending the life expectancy of cancer patients is just as fundamental as considering their quality of life. ${ }^{1}$

One of the key objectives in patients' cancer care is improving their quality of life by implementing a multidisciplinary therapeutic approach. It is now a widespread opinion in the medical community that it is necessary to treat the patient in all aspects of the illness. The most important oncology societies, the American Society of Clinical Oncology (ASCO) and the European Society for Medical Oncology (ESMO), have established the importance of an integral approach to patients' illness and more specifically, treating it from a biological, psychological, and sociological point of view.

Aside from alterations in the endocrine, cardiovascular and neurological systems, the burden of cancer and its treatment can also lead to changes in the patients' physical appearance. In fact, patients with cancer or a history of cancer often experience discomfort with their own image due to scarring, disfiguring surgeries, hair loss, or weight loss/gain. In women with breast cancer, it has been identified that changes in physical appearance are among the most significant causes of 
distress. Therefore, it becomes imperative to pay more attention to a patients' body image in order to improve their quality of life. ${ }^{2}$

Dermatologists are currently playing a more prominent role, actively taking measures to prevent and treat any therapy-related adverse events, as well as preserving patients' self-image by avoiding stigmatisation and any reminder of the disease. Stimulating a positive self-image for the patient undoubtedly has a beneficial impact on selfesteem, mental state and personal relationships, thus improving the response to any treatment and prognosis. In this regard, the demand for aesthetic treatments among cancer patients is continuously growing. These aesthetic treatments are generally safe; however, it must be made clear that in most medical treatments there is always a possibility that adverse events may occur. ${ }^{3}$

Particular attention must be paid to the cancer patient who is by definition considered "fragile". The main purpose of this review is to evaluate the safety of several aesthetic treatments, for example: fillers, botulinum toxin and laser use in cancer patients.

\section{The Cancer Patient}

In 2018, approximately 4 million new cases of cancer were estimated in Europe. ${ }^{4}$ The most frequent cancer in men is prostate cancer, whilst in women it is breast cancer. ${ }^{5}$ Cancer incidence is constantly increasing, yet cancer mortality is actually decreasing. ${ }^{6}$

In Italy, approximately $5 \%$ of the population has been diagnosed with cancer and $3 \%$ of those cases were "longsurviving" patients, whose cancer was diagnosed more than 5 years ago. About $27 \%$ of people who survive their cancer diagnosis have a similar life expectancy to those who have never received such diagnosis and by consequence, can be officially defined as "cured".

Compared to 1992, the number of Italian cancer survivors has almost doubled due to the increase in new cases (attributable to the ageing population), the higher incidence of certain cancers, and the prolonged survival after the disease. ${ }^{7}$

The term "cancer survivor" encompasses various situations, including people who are disease-free after treatment, people who continue to receive treatment in order to reduce the risk of cancer recurrence (also called adjuvant therapy) and lastly, people with a well controlled disease and minimal symptoms receiving treatment to keep the cancer under control, which often displays itself as a chronic disease.
However, a cancer survivor is considerably more fragile than a person who has never experienced this condition. The impact of a tumor and its treatments can exist for a long time, causing a greater susceptibility to infections, alterations in immune response, or an aggravation of pre-existing conditions such as diabetes or heart disease.

In case of alterations in the immune system in cancer patients, the tumor is able to modify the normal immune response to its advantage, by promoting the production of pro-inflammatory cytokines and the accumulation of suppressive cell populations that inhibit the immune response (regulatory T-cells and myeloid-derived suppressor cells). ${ }^{8}$ Moreover, the stress associated with a cancer diagnosis and its treatment can greatly impact the immune system. In this regard, chronic cancerrelated stress suppresses the protective immune response in two important ways; firstly, through the increase of mediators such as catecholamines and glucocorticoids and secondly, by inhibiting the cell-mediated immune response, the antibody response, and the activity of natural killer (NK) cells. ${ }^{9}$ It goes beyond any doubt that both chemotherapy and target therapy can lead to immunosuppression. ${ }^{10}$

In addition, cancer patients are more susceptible to infections such as bacterial, viral, and fungal infections. The main risk factors consist of skin and mucous barrier alterations, neutropenia and, as mentioned before, a certain degree of immunosuppression. The skin and mucous barrier, that represent the first defence against infections, are most often compromised in cancer patients due to invasive procedures (eg catheters, surgery) or treatments (eg chemotherapy, radiotherapy and target therapy). ${ }^{10}$

It has been seen in many cancer patients that chemotherapy produces several changes in skin composition, causing a decrease in the sebum content, water content and TEWL (trans epidermal water loss), thus compromising skin barrier function. ${ }^{11}$ Radiotherapy has also been known to damage barrier function as it induces necrosis and apoptosis of the epidermal cells, consequently reducing the production of natural moisturising factors and intercellular lipids. Radiation also causes an alkalinisation of the stratum corneum, which favours bacterial and fungal proliferation. ${ }^{12}$

It must be added that cancer patients are often elderly and therefore have other comorbidities such as malnutrition, kidney failure, heart disease or diabetes which contribute to their frailty. 
Table I Aesthetic Treatment in Cancer Patients

\begin{tabular}{|l|l|}
\hline Fillers & $\begin{array}{l}\text { Hyaluronic acid (HA) } \\
\text { Methylcellulose } \\
\text { Calcium hydroxyapatite (CHA) } \\
\text { Permanent materials }\end{array}$ \\
\hline Botulinum neurotoxins (BoNTs) & $\begin{array}{l}\text { Type A (BoNT-A) } \\
\text { Type B (BoNT-B) }\end{array}$ \\
\hline Laser therapies & $\begin{array}{l}\text { Vascular } \\
\text { Depigmentation } \\
\text { Depilatory } \\
\text { Resurfacing lasers }\end{array}$ \\
\hline
\end{tabular}

\section{Aesthetic Medicine in Cancer Patients}

Aesthetic treatments are increasingly in demand, particularly in western countries, these can be divided into treatments such as fillers, botulinum toxin or laser therapy (Table 1).

Dermatological consequences are among the greatest stresses in cancer patients because they are a persistent sign of cancer (Table 2). Among the dermatological sequelae, the main ones are surgical scars, striae distensae, persistent alopecia, hirsutism, telangiectasias, radiation tattoos, lymphoedema, premature ageing and volume loss. ${ }^{13}$ Given the strong impact these manifestations can have on quality of life, it is important to evaluate therapies that can

Table 2 Most Frequent Cancer Treatment Sequelae

\begin{tabular}{|l|l|}
\hline Treatment & \multicolumn{1}{|c|}{ Clinical Sequelae } \\
\hline Surgery & $\begin{array}{l}\text { Scarring, fibrosis, keloids lymphoedema, } \\
\text { hypertrichosis }\end{array}$ \\
\hline Radiotherapy & $\begin{array}{l}\text { Alopecia, fibrosis, telangiectasia, lymphoedema, } \\
\text { hyper/hypopigmentation, radiation tattoo }\end{array}$ \\
\hline $\begin{array}{l}\text { Chemotherapy } \\
\text { agents }\end{array}$ & $\begin{array}{l}\text { Alopecia, hyperpigmentation, nails changes } \\
\text { (onycholysis, onychomadesis, hyperpigmentation, } \\
\text { permanent dystrophy, iatrogenic hirsutism, } \\
\text { hypertrichosis, paronychia, brittle nails, pyogenic } \\
\text { granuloma-like lesions, Beau's lines), paronychia } \\
\text { telangiectasia, striae, vitiligo } \\
\text { Alopecia, hair changes (depigmentation, brittle } \\
\text { hair), hyperpigmentation, nails changes } \\
\text { agents }\end{array}$ \\
$\begin{array}{l}\text { (onycholysis, onychomadesis, hyperpigmentation, } \\
\text { permanent dystrophy, iatrogenic hirsutism, } \\
\text { hypertrichosis, paronychia, brittle nails, pyogenic } \\
\text { granuloma-like lesions, Beau's lines, leukonychia), } \\
\text { photosensitivity, paronychia, xerosis, oedema }\end{array}$ \\
\hline
\end{tabular}

treat them. To date, little is known about the use of aesthetic treatments in cancer patients.

\section{Fillers}

There are different types of fillers, the most commonly used are: hyaluronic acid (HA), methylcellulose, calcium hydroxyapatite (CHA) and permanent materials.

In cancer patients, fillers can be used to correct volume loss that can occur in the upper third of the face as consequence of the disease, or to improve the aesthetic result of post-surgery scars. ${ }^{14}$ Moreover, the use of fillers is approved in cancer patients to prevent skin toxicity, secondary to some therapies such as radiotherapy, and have also been implied as a reconstructive technique, particularly for breast cancer. ${ }^{15}$

Although generally safe, filler injections can sometimes cause adverse events which can be distinguished as reactions at the site of injection or an inflammatory, vascular reaction. Injection site reactions include erythema, oedema, pain and bruising. ${ }^{16}$ Filler injections can cause both immediate localised reactions, or systemic hypersensitivity reactions (fever and urticaria) and delayed hypersensitivity reactions with the formation of granulomatous bodies. As a result of penetration into the skin barrier, fillers may be associated with an increased risk of infection; reactivation of herpes simplex infections have frequently been described and can be prevented by prophylactic antiviral therapy. Among bacterial infections, the most frequent are those caused by Staphylococci and Streptococci such as cellulitis and abscesses. Moreover, even after a long time, the formation of biofilms is possible and can predispose patients to bacterial infections, nodule formation and granulomatous reactions. ${ }^{3}$

\section{Fillers in Cancer Patients}

In the treatment of cancer patients, it is necessary to adopt the same precautions we use for other patients whilst paying particular attention to the presence of immunesuppression, the persistence of skin complications, and interactions with existing therapies. Given the increased risk of infections in these patients, it will be necessary to ensure maximum asepsis.

The most suitable material to be used is autologous fat, even if other types of filler are also considered to be safe. To the best of our knowledge, there is limited literature regarding the safety and efficacy of these injection treatments. However, we have identified a Phase 4 study conducted in women with breast cancer where the use of HA 
did not lead to adverse events. ${ }^{17}$ Moreover, HA filler injections have also been used successfully in a chronic myeloid leukemia patient taking imatinib mesylate. ${ }^{18}$

However, it must be kept in mind that some fillers can create interpretation problems when performing instrumental examinations, for example CHA can appear with a similar appearance to malignant formations on computed tomography (CT), fluorodeoxyglucose-positron emission tomography (FDG-PET) and magnetic resonance imaging (MRI).

The main contraindication to filler injections is represented by patients without a good general condition, for example: have ongoing infections, are not immunocompetent, are undergoing treatment and have not received approval from their oncologist. Particular attention should be paid to patients who underwent therapies with bisphosphonates, avoiding injections into the bone compartment given the high risk of osteonecrosis.

The description of granulomatous reactions in patients receiving immunotherapy deserves a separate discussion. Foreign body reactions occur between $0.04-0.3 \%$ of filler injection cases. Among the risk factors for developing such reactions are the use of immunotherapy drugs. ${ }^{19}$

First-line therapy is represented by intralesional injections of corticosteroids, possibly followed by systemic therapy in the relapsing forms. Several cases of granulomatous reactions have been described in patients treated with immunotherapy (anti PD1 or anti CTLA4), who had previously received filler injections many years before. $^{20,21}$ Check-point inhibitors appear to act as a trigger for the formation of foreign bodies against fillers. To the best of our knowledge, only one case of a granulomatous/sarcoid-like reaction has been described in patients receiving anti-BRAF therapy. Dermal fillers may induce late-onset adverse skin reactions in patients under BRAF inhibitors.

Therefore, it is very important that oncologists and dermatologists are aware of these reactions when evaluating the onset of nodules in patients undergoing immunotherapy. ${ }^{21}$

\section{Botulinum Toxin}

Botulinum neurotoxins (BoNTs) are produced by various strains of Clostridium botulinum, which are gram-positive, obligate anaerobic bacteria. Two serotypes, type A (BoNTA) and type B (BoNT-B), are currently in clinical use. ${ }^{22}$ In clinical practice, BoNTs are used for numerous approved and off-label indications. BoNT causes muscle paralysis by inhibiting the release of acetylcholine from the presynaptic terminal. After a BoNT injection, the first clinical effects usually occur few days to 4-6 weeks later. The duration of the effects usually last up to 10-12 weeks but can change according to the dose, the severity, and the individual characteristics of the patient. ${ }^{22}$

\section{Botulinum Toxin Indications, Safety, and Complication}

BoNT injection is approved for treatment of blepharospasm, glabellar frown lines in adults, primary axillary hyperhidrosis, strabismus, hemifacial spasm, cranial nerve VII disorders, cervical dystonia, upper limb spasticity, prophylaxis for chronic migraine and bladder hyperactivity. ${ }^{23,24}$ Off-label uses are represented by wrinkle reduction in the neck, chest, upper/lower face, correction of facial asymmetries and lifting of facial areas. ${ }^{25}$

BoNT have an excellent safety profile when used in minute quantities by experienced clinicians. However, some side effects have been described, particularly complications at the injection site such as erythema, oedema, bruising and pain. ${ }^{3,26}$ Urticaria, anaphylactic reactions and dyspnea can occur rarely.

Depending on the injection site, other complications may include lip ptosis and Brown syndrome with consequent asymmetries. In the treatment of neck blemishes, an overdose or overly deep injection can cause xerostomia, dysphagia, dysarthria, and neck weakness. ${ }^{3}$

\section{Botulinum Toxin Resistance}

In some patients BoNT has limited benefits, resulting in a primary non-response when resistance occurs after the first injection, and a secondary non-response when there is a loss of efficacy after subsequent injections. ${ }^{22}$

In a study conducted on 235 patients treated with BoNT for various indications, the primary non-response rate was $9.1 \%$ and the secondary non-response rate was $7.5 \%{ }^{27-29}$ Primary non-responders (PNR) are generally patients who have received an insufficient dose of toxin or who have previously been vaccinated against the botulinum toxin. Furthermore, antibodies could also be found in patients previously affected by botulism. ${ }^{30}$ On the other hand, the secondary non-response seems to be due to the formation of neutralising antibodies directed against the fundamental epitope of BoNT or its binding region. BoNT's protein acts as an antigen that can stimulate the patient's immune response and therefore, the formation of 
antibodies. Amongst the main factors that seem to favour antibody formation are: closer administration intervals, excessive dose injected per cycle, and a large amount of antigenic protein. ${ }^{31,32}$

\section{Botulinum Toxin in Cancer Patients}

To our knowledge, little is known about the use of botulinum toxin in cancer patients to date. However, BoNT injections could be useful for treating conditions caused by cancer and the consequences of its treatment (surgery, chemotherapy, radiotherapy) such as asymmetries, functional alterations, premature ageing, spasticity, hyperhidrosis, dyskinesias and pain.

The injection of botulinum toxin is rarely offered as a possibility to cancer patients due to the fear of complications. However, this procedure has proven to be safe in this category of patients. ${ }^{17}$ For example, it has been shown to be effective and safe for post-surgical and postradiotherapy pain in: the management and prevention of surgical complications in patients with head and neck cancer, ${ }^{33}$ the treatment of anal hypertonia in patients treated with chemotherapy ${ }^{34}$ and, the prevention of glandular function in head and neck cancer patients treated with radiotherapy. ${ }^{35}$

It is important to consider the increased risk of BoNT neutralising antibody formation in cancer patients treated with monoclonal antibodies, especially in the case of immunotherapeutic drugs. In fact, monoclonal antibodies induce the activation of B lymphocytes and plasma cells against their own epitopes with consequent antibody production, which could cross-react with the proteins expressed by botulinum toxin. ${ }^{36}$ Immunotherapy drugs could further fuel this process, similar to the enhancement process of the host's immune response however, in one case of a melanoma patient, the toxin was used effectively and safely to treat Ipilimumab-induced peripheral vasculitis.

Particular attention must be paid to the use of BoNT in patients suffering from thymoma. In fact, myasthenia gravis is the most common paraneoplastic syndrome diagnosed in these patients. ${ }^{37}$

It is known that the toxin is contraindicated in patients suffering from neuromuscular diseases such as myasthenia gravis, amyotrophic lateral sclerosis and Eaton Lambert syndrome. $^{38}$

It is also interesting to mention that the role of botulinum toxin injections has been evaluated in increasing the efficacy of radiotherapy and chemotherapy treatments; toxin-induced vasodilation increases the tumor's response to these treatments. ${ }^{39}$

\section{Laser Therapy}

Four categories of lasers are used in dermatology: vascular, depigmentation, depilatory, and resurfacing lasers. In cancer patients, each of these laser categories are widely used for treatment of dermatological sequelae. The safety and efficacy of vascular lasers and light therapy [Pulse Dye Laser (PDL) or Intense Pulsed Light (IPL)] in the treatment of post-radiotherapy telangiectasias has been demonstrated. $^{40}$ In particular, PDL proved to be more effective and less painful. In a study conducted in women with breast cancer, the use of PDL to treat dermatitis from radiotherapy has been shown to improve patients' quality of life. ${ }^{41}$ Obviously in an oncology patient, it is necessary to pay close attention, avoiding the use of lasers during the course of acute dermatitis and carefully evaluating the state of the patient's skin. Both vascular lasers and fractional ablative lasers can be used in the treatment of fibrosis induced by radiotherapy or associated with graft versus host disease (GVHD). ${ }^{42}$ Even radiotherapy tattoos can often cause a negative impact on patient's quality of life by being a continual reminder of past illness. The ablative laser in particular has been used successfully in the elimination of these radiotherapy tattoos.

Depilatory lasers (Long Pulsed Alexandrite, Nd: YAG laser, Diode laser) are indicated for the treatment of hypertrichosis and hirsutism conditions, mostly secondary to therapy with an epidermal growth factor receptor (EGFR) inhibitor. ${ }^{43}$ In fact, shaving or waxing can be more aggressive on the sensitive skin of cancer patients.

In cancer patients, the use of lasers is associated with two main problems: the risk of photosensitive reactions, and infection (REF).

\section{Conclusions}

This review has shown that cancer patients are fragile patients, from both a physical and an emotional point of view. Therefore, the doctor's role must be to treat the patient whilst considering all of these different points of view. Changes in physical appearance induced by cancer and its treatments are some of the greatest sources of stress for patients, therefore, it is essential to evaluate the safety of aesthetic treatments that we can offer them. Particularly in the use of fillers, it will be essential to consider the possible interference of materials used with instrumental 
diagnostic techniques. Therefore, it will be important for the patient to report every possible consequence of the aesthetic procedure to their oncologist. For the oncologist, it is fundamental to always analyses the patient's personal history carefully, and whether or not there is a possible use of these treatments. In addition, when using fillers, particular attention must be paid to the concomitant therapy of the patient, given the increased risk of granulomatous reactions in patients treated with immuno-checkpoints. When using botulinum toxin, particular attention should be paid to avoid treatment in patients suffering from neuromuscular paraneoplastic syndromes. In addition, the possible lower benefit/efficacy in patients treated with monoclonal antibodies (target therapy/immunotherapy) should be considered, given the increased risk of antidrug antibody formation. However, in the use of lasers, the risk of phototoxic and photoallergic reactions must be considered and their use in patients receiving photosensitising drugs avoided.

In the case of all filler, botulinum toxin and laser treatments, given the possible alterations in the cancer patient's skin barrier, and in many cases the presence of already compromised skin, it will be essential to ensure maximum aseptic conditions, and eventually carry out antibiotic/antiviral prophylactic therapies to reduce the risk of infections.

Finally, it is important to point out that the aesthetic doctor/dermatologist within the multidisciplinary team, who meticulously takes care of the cancer patient, has a central role. This person will have to carefully consider not only the most appropriate procedures for each patient along with preventing and treating any adverse events related to the therapies carried out but must also be able to recognise early signs of a possible disease reactivation.

\section{Acknowledgments}

The authors thank Content Ed Net for editorial support, with the helpful contribution of medical writer Giovanna Damia, M.D.

\section{Author Contributions}

All authors made a significant contribution to the work reported, whether that is in the conception, study design, execution, acquisition of data, analysis and interpretation, or in all these areas; IP and CP took part in drafting the article; NS, AM, FM, ET, NB, AM, GR and SV took part in revising the article; all authors gave final approval of the version to be published; all authors have agreed on the journal to which the article has been submitted and all authors agree to be accountable for all aspects of the work.

\section{Funding}

Medical writing assistance was funded by Novartis Farma Italy.

\section{Disclosure}

The authors declare no conflicts of interest in this work.

\section{References}

1. Firkins J, Hansen L, Driessnack M, Dieckmann N. Quality of life in "chronic" cancer survivors: a meta-analysis. $J$ Cancer Surviv. 2020;14(4):504-517. doi:10.1007/s11764-020-00869-9

2. Hopwood P, Fletcher I, Lee A, Al Ghazal S. A body image scale for use with cancer patients. Eur J Cancer. 2001;37(2):189-197. doi:10.1016/S0959-8049(00)00353-1

3. Kassir M, Gupta M, Galadari H, et al. Complications of botulinum toxin and fillers: a narrative review. $J$ Cosmet Dermatol. 2020;19 (3):570-573. doi:10.1111/jocd.13266

4. Ferlay J, Colombet M, Soerjomataram I, et al. Cancer incidence and mortality patterns in Europe: estimates for 40 countries and 25 major cancers in 2018. Eur $J$ Cancer. 2018;103:356-387. doi:10.1016/j. ejca.2018.07.005

5. Torre LA, Siegel RL, Ward EM, Jemal A. Global cancer incidence and mortality rates and trends-an update. Cancer Epidemiol Biomarkers Prev. 2016;25(1):16-27. doi:10.1158/1055-9965.EPI-15-0578

6. Buzzoni C, Crocetti E, Guzzinati S, Dal Maso L, Francisci S, Group AW. Cancer incidence and mortality trends from 2003 to 2014 in Italy. Tumori. 2019;105(2):121-137. doi:10.1177/ 0300891619839844

7. (AIRTUM) AIRT. I tumori in Italia - Rapporto AIRTUM 2014. Prevalenza e guarigione da tumore in Italia. Epidemiol Prev. 2014;38(6Suppl 1):1-44.

8. Finn OJ. Immuno-oncology: understanding the function and dysfunction of the immune system in cancer. Ann Oncol. 2012;23(Suppl 8): viii6-viii9. doi:10.1093/annonc/mds256

9. Antoni MH, Dhabhar FS. The impact of psychosocial stress and stress management on immune responses in patients with cancer. Cancer. 2019;125(9):1417-1431. doi:10.1002/cncr.31943

10. Angarone M. Fungal infections in cancer patients. Cancer Treat Res. 2014;161:129-155.

11. Kang D, Kim IR, Im YH, et al. Quantitative changes in skin composition parameters due to chemotherapy in breast cancer patients: a cohort study. Breast Cancer Res Treat. 2015;152(3):675-682. doi:10.1007/s10549-015-3502-4

12. Hu SC, Hou MF, Luo KH, et al. Changes in biophysical properties of the skin following radiotherapy for breast cancer. $J$ Dermatol. 2014;41(12):1087-1094. doi:10.1111/1346-8138.12669

13. Rossi AM, Hibler BP, Navarrete-Dechent C, Lacouture ME. Restorative oncodermatology: diagnosis and management of dermatologic sequelae from cancer therapies. $J$ Am Acad Dermatol. 2021;85(3):693-707.

14. Kasper DA, Cohen JL, Saxena A, Morganroth GS. Fillers for postsurgical depressed scars after skin cancer reconstruction. $J$ Drugs Dermatol. 2008;7(5):486-487.

15. Struik GM, Godart J, Verduijn GM, et al. A randomized controlled trial testing a hyaluronic acid spacer injection for skin toxicity reduction of brachytherapy accelerated partial breast irradiation (APBI): a study protocol. Trials. 2018;19(1):689. doi:10.1186/s13063-0183035-3 
16. Galadari H, Krompouzos G, Kassir M, et al. Complication of soft tissue fillers: prevention and management review. J Drugs Dermatol. 2020;19(9):829-832. doi:10.36849/JDD.2020.5084

17. Shamban A. Safety and efficacy of facial rejuvenation with small gel particle hyaluronic acid with lidocaine and A botulinum toxin A in post-chemotherapy patients: a Phase IV Investigator-initiated Study. $J$ Clin Aesthet Dermatol. 2014;7(1):31-36.

18. Sung Y, Kim MH, Cho E. Successful hyaluronic acid filler injection in a chronic myeloid leukemia patient taking imatinib mesylate. J Cosmet Laser Ther. 2019;21(4):243-244. doi:10.1080/147641 72.2018.1525745

19. Dhaliwal P, Ibad S, Losak D, Cockerell C. A case of granulomatous hypersensitivity reactions to a dermal filler precipitated by PD-1 checkpoint inhibitor therapy. SKIN J Cutan Med. 2021;5(1):41-45. doi:10.25251/skin.5.1.10

20. Bisschop C, Bruijn MS, Stenekes MW, Diercks GF, Hospers GA. Foreign body reaction triggered by cytotoxic $T$ lymphocyte-associated protein 4 blockade 25 years after dermal filler injection. $\mathrm{Br} J$ Dermatol. 2016;175(6):1351-1353. doi:10.1111/ bjd. 14674

21. Syunyaeva Z, Kahnert K, Kauffmann-Guerrero D, Huber RM, Tufman A. Dermal filler injections mimic tumor activity during immune checkpoint inhibition. Respiration. 2018;95(5):362-363. doi:10.1159/000487601

22. Jankovic J. Botulinum toxin: state of the art. Mov Disord. 2017;32 (8):1131-1138. doi:10.1002/mds. 27072

23. Dressler D. Therapeutically relevant features of botulinum toxin drugs. Toxicon. 2020;175:64-68. doi:10.1016/j.toxicon.2019.12.005

24. Zalvan C, Bentsianov B, Gonzalez-Yanes O, Blitzer A. Noncosmetic uses of botulinum toxin. Dermatol Clin. 2004;22(2):187-195. doi:10.1016/S0733-8635(03)00020-2

25. Sundaram H, Signorini M, Liew S, et al. Global aesthetics consensus: botulinum toxin type A-evidence-based review, emerging concepts, and consensus recommendations for aesthetic use, including updates on complications. Plast Reconstr Surg. 2016;137(3):518e-529e. doi:10.1097/01.prs.0000475758.63709.23

26. Kroumpouzos G, Kassir M, Gupta M, Patil A, Goldust M. Complications of botulinum toxin A: an update review. $J$ Cosmet Dermatol. 2021;20(6):1585-1590. doi:10.1111/jocd.14160

27. Fischer T, Sattler G, Prager W, et al. Safety, tolerability, and efficacy of repeat-dose injections of incobotulinumtoxinA in the treatment of upper facial lines: results from a Prospective, Open-Label, Phase III Study. J Drugs Dermatol. 2020;19(5):461-469. doi:10.36849/ JDD.2020.5013

28. Rzany B, Ascher B, Monheit G. Treatment of glabellar lines with botulinum toxin type A (speywood unit): a clinical overview. $J$ Eur Acad Dermatol Venereol. 2010;24(Suppl 1):1-14. doi:10.1111/ j.1468-3083.2009.03475.x

29. Lawrence I, Moy R. An evaluation of neutralizing antibody induction during treatment of glabellar lines with a new US formulation of botulinum neurotoxin type A. Aesthet Surg J. 2009;29(6 Suppl):S66S71. doi:10.1016/j.asj.2009.09.009
30. Stephan F, Habre M, Tomb R. Clinical resistance to three types of botulinum toxin type A in aesthetic medicine. $J$ Cosmet Dermatol. 2014;13(4):346-348. doi:10.1111/jocd.12108

31. Bellows S, Jankovic J. Immunogenicity associated with botulinum toxin treatment. Toxins. 2019;11(9):491. doi:10.3390/toxins11090491

32. Naumann M, Boo LM, Ackerman AH, Gallagher CJ Immunogenicity of botulinum toxins. J Neural Transm. 2013;120 (2):275-290. doi:10.1007/s00702-012-0893-9

33. Shaw L, Bazzell AF, Dains JE. Botulinum toxin for side-effect management and prevention of surgical complications in patients treated for head and neck cancers and esophageal cancer. $J A d v$ Pract Oncol. 2019;10(1):40-52.

34. Roelandt P, Coremans G, Wyndaele J. Analgesic injection of botulinum toxin in anal fissures is efficient and can be performed safely in patients actively receiving chemotherapy. Support Care Cancer. 2020;28(11):5053-5054. doi:10.1007/s00520-020-05641-4

35. Teymoortash A, Pfestroff A, Wittig A, et al. Safety and efficacy of botulinum toxin to preserve gland function after radiotherapy in patients with head and neck cancer: a prospective, randomized, placebo-controlled, double-blinded phase I clinical trial. PLoS One. 2016;11(3):e0151316. doi:10.1371/journal.pone.0151316

36. Talotta R, Rucci F, Canti G, Scaglione F. Pros and cons of the immunogenicity of monoclonal antibodies in cancer treatment: a lesson from autoimmune diseases. Immunotherapy. 2019;11 (3):241-254. doi:10.2217/imt-2018-0081

37. Tormoehlen LM, Pascuzzi RM. Thymoma, myasthenia gravis, and other paraneoplastic syndromes. Hematol Oncol Clin North Am. 2008;22(3):509-526. doi:10.1016/j.hoc.2008.03.004

38. Dayan SH, Bassichis BA. Evaluation of the patient for cosmetic botox injections. Facial Plast Surg Clin North Am. 2003;11 (3):349-358. doi:10.1016/S1064-7406(03)00022-1

39. Ansiaux R, Baudelet C, Cron GO, et al. Botulinum toxin potentiates cancer radiotherapy and chemotherapy. Clin Cancer Res. 2006;12 (4):1276-1283. doi:10.1158/1078-0432.CCR-05-1222

40. Nymann P, Hedelund L, Haedersdal M. Intense pulsed light vs. long-pulsed dye laser treatment of telangiectasia after radiotherapy for breast cancer: a randomized split-lesion trial of two different treatments. Br J Dermatol. 2009;160(6):1237-1241. doi:10.1111/ j.1365-2133.2009.09104.x

41. Rossi AM, Blank NR, Nehal K, Dusza S, Lee EH. Effect of laser therapy on quality of life in patients with radiation-induced breast telangiectasias. Lasers Surg Med. 2018;50(4):284-290. doi:10.1002/ 1sm. 22780

42. Labadie JG, Kosche C, Kyllo R, et al. Fractional CO2 laser for the treatment of sclerodermatous cGVHD. J Cosmet Laser Ther. 2020;22 (1):49-51. doi:10.1080/14764172.2019.1710537

43. Gan SD, Graber EM. Laser hair removal: a review. Dermatol Surg. 2013;39(6):823-838. doi:10.1111/dsu.12116
Clinical, Cosmetic and Investigational Dermatology

\section{Publish your work in this journal}

Clinical, Cosmetic and Investigational Dermatology is an international, peer-reviewed, open access, online journal that focuses on the latest clinical and experimental research in all aspects of skin disease and cosmetic interventions. This journal is indexed on CAS
The manuscript management system is completely online and includes a very quick and fair peer-review system, which is all easy to use. Visit http://www.dovepress.com/testimonials.php to read real quotes from published authors. 